\title{
Silicon photonics biosensing: different packaging platforms and applications
}

\author{
C. Lerma Arce ${ }^{\mathrm{a}, \mathrm{b}}$, E. Hallynck ${ }^{\mathrm{a}, \mathrm{b}}$, S. Werquin ${ }^{\mathrm{a}, \mathrm{b}}$, J.W. Hoste ${ }^{\mathrm{a}, \mathrm{b}}$, D. Martens ${ }^{\mathrm{a}, \mathrm{b}}$, P. Bienstman ${ }^{\mathrm{a}, \mathrm{b},{ }^{*}}$ \\ ${ }^{a}$ Photonics Research Group (INTEC) Ghent University, Sint-Pietersnieuwstraat 41, 9000 Gent, \\ Belgium; ${ }^{\mathrm{b} C e n t e r}$ for Nano- and Biophotonics, Ghent University, 9000 Ghent, Belgium
}

\begin{abstract}
We present two different platforms integrating silicon photonic biosensors. One is based on integration with reaction tubes to be compatible with traditional lab approaches. The other uses through-chip fluidics in order to achieve better mixing of the analyte.
\end{abstract}

Keywords: biosensors, silicon photonics, reaction tubes, flow-through microfluidics

\section{REACTION TUBES AS A PLATFORM FOR RING RESONATOR SENSORS}

\subsection{Introduction}

Label-free biosensing with silicon nanophotonic microring resonator sensors has proven to be an excellent technique for achieving high-throughput and high sensitivity, comparing favorably with other labeled and label-free sensing techniques. However, as in any biosensing platform, silicon nanophotonic microring resonator sensors require a fluidic component which allows the continuous delivery of the sample to the sensor surface. This is the big disadvantage of this platform since this type of microfluidic system is very much removed from the daily practice in e.g. hospital labs, which still relies to a large degree on platforms like 96-well microtiter plates, or reaction tubes.

Here we propose the combination of a simple and lab-compatible reaction tube platform, with label-free nanophotonic biosensors with a special microfluidic system imbedded in the same chip, where the flow is through the chip as opposed to over the chip as in more traditional approaches. This shows that label-free nanophotonic ring resonators can be also used in the user-friendly platform like reaction tubes or well microtiter plates, conserving their excellent performance.

\section{Nanophotonic ring resonator sensors, chip layout, and setup}

The photonic chip was fabricated in SOI with 2- $\mu \mathrm{m}$ buried oxide and a 220-nm silicon top layer with CMOS compatible 193-nm optical lithography and dry etching [1]. The ring resonators consist of 450-nm-wide single-mode waveguides, with $5-\mu \mathrm{m}$ bend radius, $2-\mu \mathrm{m}$-long directional couplers, and a gap of $180 \mathrm{~nm}$ between the waveguides. The layout of the chip can be seen in Figure 1.
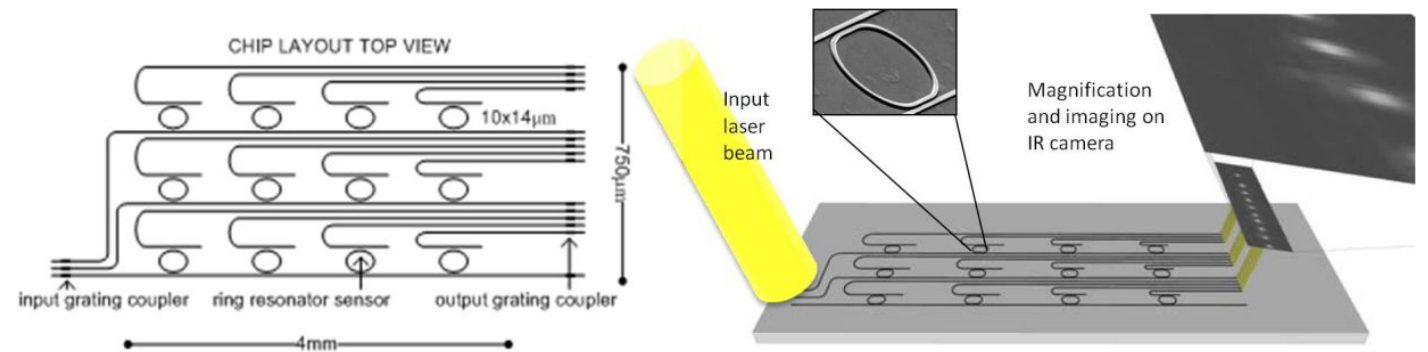

Figure 1. Left. Chip layout top view. Four rings are connected to one common input waveguide, each of them having a dedicated drop signal port. Three of these four ring series are placed independently next to the other. Right: Setup: A SANTEC TSL-510 tunable laser was used as a light source. The output signals of the ring resonators are imaged with an infrared camera [2]. 
Four rings are connected to one common input waveguide, each of them having a dedicated drop signal port. Three of these four ring series are placed independently next to the other. The three input waveguides are simultaneously addressed through vertical grating couplers [3] with a 2-mm-wide collimated beam from a tunable laser source. The output signals of the ring resonators are near-vertically coupled to free space by means of integrated grating couplers and are imaged with an infrared camera.

A TSL-510 tunable laser is used as a light source. The transmitted light is detected by an infrared camera. We have developed software that captures an image for every wavelength step and stores the maximum intensity values within each dedicated area that overlaps with an output grating coupler spot. Post-processing consists of fitting the spectra to their theoretical shape and tracking these resonance peaks over time. This optical setup allows very high alignment tolerances, measures the spectrum of all the ring resonators in parallel, and therefore presents no limitation for highthroughput sensing.

\section{Embedded microfluidic system}

We propose a microfluidic system embedded in the photonic chip that will be integrated at the bottom of the reaction tube. This microfluidic system consists of certain apertures that perforate the chip from the top to the bottom. The solution inserted in the tube will flow through these openings that work as exit channels, creating a flow, which will accelerate the detection process. Figure 2 shows a schematic of the device with the embedded microfluidic system.

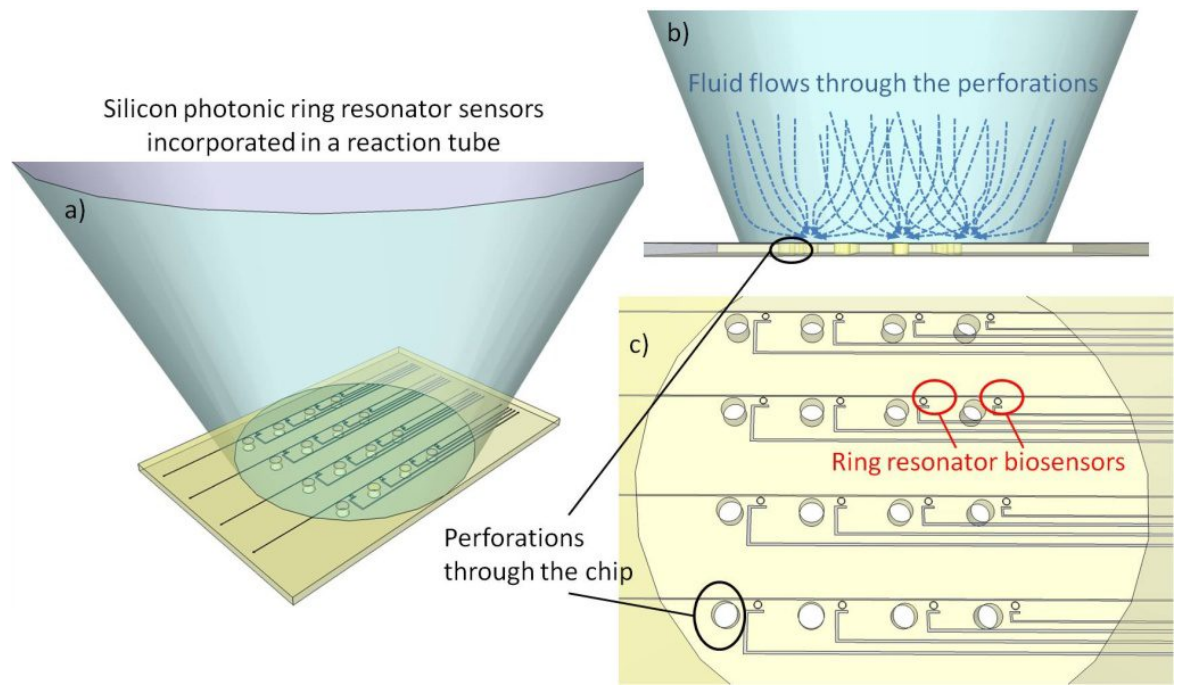

Figure 2. a) Schematic of the device. The silicon-on-insulator chip with the photonic biosensors and the embedded microfluidic system is incorporated at the bottom of a reaction tube. b) The solution inserted in the tube will flow through these openings that work as exit channels, creating a flow, which will accelerate the detection process. c) Layout of the chip. The array of ring resonators sensors is accompanied by an array of perforations next to them.

A Duetto laser source (Time-Bandwidth) was used to perform the perforations of the silicon-on-insulator chip by means of laser ablation.

\section{Experiments and results}

The photonic chip with the array of sensors and the embedded microfluidic system described above is incorporated to the bottom of the reaction tube, once its original bottom is mechanically removed. The attachment of the chip to the bottom of the tube is done permanently using UV curable glue which allows us to align precisely the array of sensors in the center of the reaction tube.

To perform the experiments, the device was fixed on a tiny chuck by means of vacuum. This chuck also has a connection to a pump, where pressure can be applied positively or negatively, pushing or sucking any gas of fluid applied in a specific area of this chuck. When the fluid under analysis will be inserted in the tube, this will be in contact with the sensors, and flow out through the holes and be sucked or pushed up again by the pump. Figure 6a shows the device fixed on this chuck.

A new aspect with respect to our previous work [2] is that now, we have the possibility of coupling light in and out from the bottom of the chip, i.e. through the silicon substrate. (Figure 6b) Silicon is considered practically transparent for 
the wavelength used $(1.55 \mu \mathrm{m})$, however a few simple processing steps need to be done in advance to facilitate the alignment and detection of light.

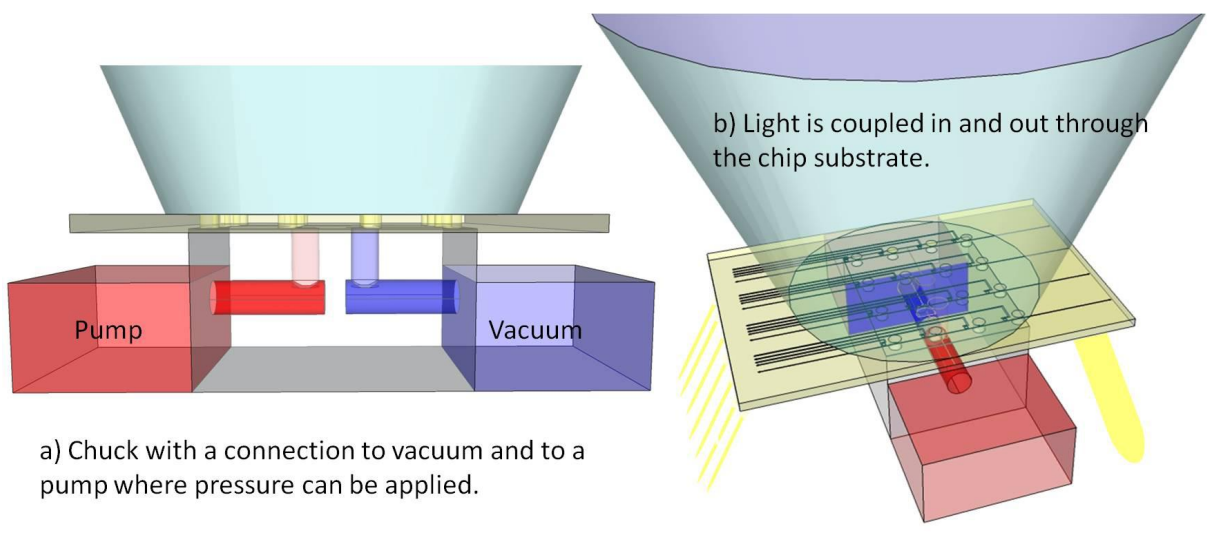

Figure 3. a) Device mounted on the chuck with a connection to vacuum and to a pump, where pressure can be applied in order to suck or push the fluid on the chip surface. b) The device is fixed to the chuck with vacuum. The measurements are performed from the bottom of the chip, i.e. light is coupled in and out through the chip substrate.

As a proof-of-principle to show the capabilities of the combined device, we measured the different steps for an assay where the well-known high-affinity couple biotin-streptavidin was bound. The measurements were performed as follows: Small volumes of different solutions were manually pipetted in the tube, and they were sucked out through the perforations of the bottom of the tube while being measured. The experiment consists of three different steps: silanization of the surface with aminosilane 3-Aminopropyl)triethoxysilane (APTES), immobilization of biotin and binding of streptavidin. Figure 8 shows three different graphs corresponding to each one of the steps of the assay. They show the evolution in time of the resonance wavelength shift of the ring resonators during the measurements with different solutions. Each color corresponds to one sensor.
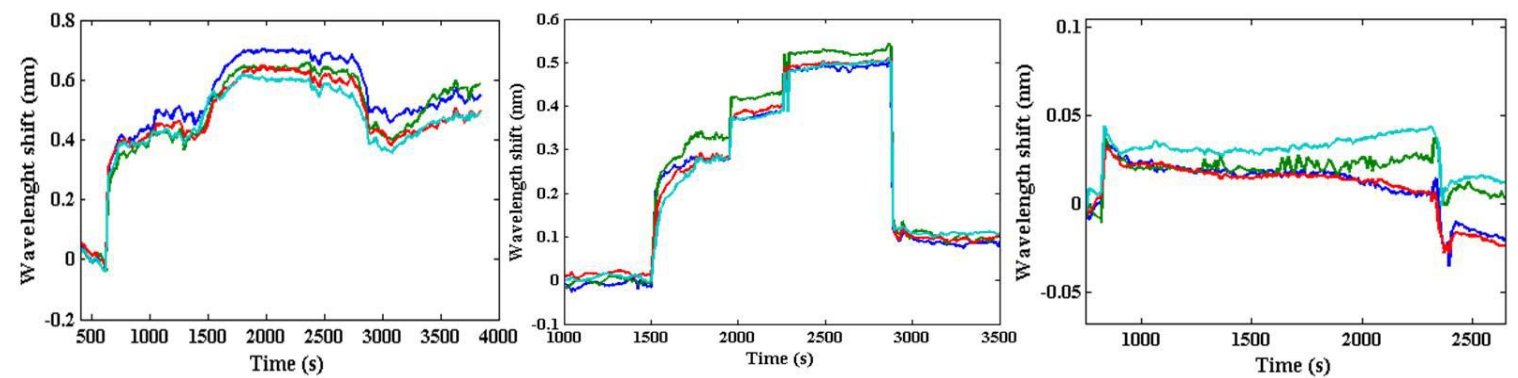

Figure 4. Three different steps of the bioassay were measured. From left to right. a) Silanization of the surface with APTES: ethanol-APTES- ethanol b) inmobilization of the biotin: PBS- biotin- PBS. c) binding of streptavidin to biotin: PBSstreptavidin- PBS

The association and disassociation of APTES in the sensor is easily quantifiable in Figure 8 Left. Figure 8 Center shows the binding of biotin after flowing $3 \mathrm{mg} / \mathrm{ml}$ solution of biotin in PBS and its disassociation when it is rinsed with PBS. A shift of $30 \mathrm{pm}$ is measured when streptavidin $(0.1 \mathrm{mg} / \mathrm{ml}$ in PBS) is flowed through the chip proving the binding of this to the biotin.

\section{DIGITAL MICROFLUIDICS WITH PRESSURE-BASED ACTUATION}

\subsection{Introduction}

In this section we will focus on a different form factor to integrate sensors, using droplets without any microfluidic channel: consider a droplet of analyte on a biosensor with holes where we want the target molecules to bind at the walls 
of the holes (see Figure 5). Gravity will not be able to sustain a flow by itself since it is counteracted by surface tension of the liquid. However, it is possible to initiate a flow by creating a pressure gradient over the droplet: creating an underpressure at the bottom of the droplet will cause the analyte containing the target molecules to be sucked through the openings. The target molecules will be brought in close contact with the receptor molecules with which they can bind. If we remove the pressure gradient, the droplet will be hanging on the other side of the membrane (i.e. the bottom). Since not all target molecules will have bonded to receptor molecules yet, we can push the droplet back through the openings by applying an overpressure at the bottom. The target molecules again pass closely to the receptor molecules and have a good chance at binding. If we stop the pressure gradient after a certain time, the droplet will be on top of the membrane once again. We can repeat this cycle several times to ensure most biomolecules have bonded in the membrane holes after some time. There is no need to encapsulate the sensor in a flow cell, saving on packaging costs and improving reliability (due to the high pressures involved in microfluidics, some flow cells tend to leak after some time).
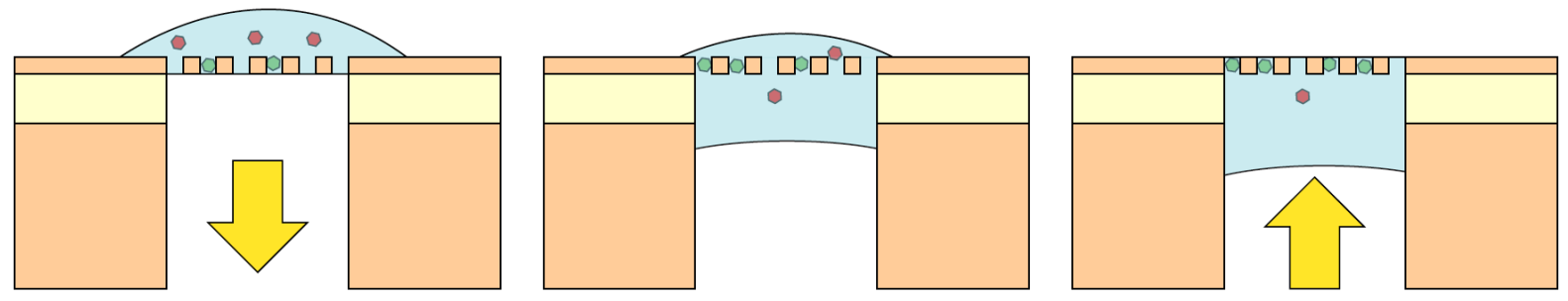

Figure 5. By applying underpressure at the bottom of the liquid droplet, the biomolecules are transported through the sensor membrane. The droplet can be pushed back by applying overpressure. This cycle can be repeated several times to ensure most biomolecules have bonded in the membrane holes.

\subsection{Fabrication}

Some of the requirements for a commercially viable biosensor is that it is low cost and capable of being produced in high volumes. A good candidate that is capable of delivering these requirements is Silicon-on-Insulator (SOI) in combination with Deep Ultraviolet (Deep UV) lithography. However, since we want to obtain thin membranes, we have to locally remove the substrate and buried oxide layer from the devices received from ePIXfab. For etching the silicon substrate we have opted for the commonly used wet anisotropic etch through $\mathrm{KOH}$; for the silicon dioxide we used vapour $\mathrm{HF}$ etch since wet HF etch could cause stiction between the waveguides.

Since the standard substrate thickness of a SOI die is 725 micron, we opted to mechanically thin down the substrate first to 250 micron; otherwise, the hard mask material for the $\mathrm{KOH}$ etching process might not last long enough but more importantly, the initial window size at the substrate size would need to be much larger (due to the anisotropic nature of the etching process). This implies that the density of several sensors on one chip (e.g. for multiplexing capabilities) has to be low since two windows in the hard mask cannot overlap. After thinning of the samples, we deposit $400 \mathrm{~nm} \mathrm{SiO} 2$ using plasma enhanced chemical vapour deposition (PECVD) at both sides and after that, $400 \mathrm{~nm}$ of PECVD silicon nitride. The layer stack at the device side acts as a protective layer for the light-guiding silicon structures in the 220-nmthin silicon top layer while the layer stack at the bottom acts as a hard mask. After lithography using backside alignment (i.e. alignment markers at the device side are used to pattern the substrate side), the hard mask is locally etched using reactive ion etching (RIE). Since PECVD deposited materials generally have a non-negligible pinhole density which could allow the $\mathrm{KOH}$ to damage our silicon structures, we require an extra protective coating at the device side. For this we use the commercially available ProTEK B3 which is designed to withstand $\mathrm{KOH}$. With the layer stack finished, we put the sample in $20 \% \mathrm{KOH}$ for 3 hours, where buried silicon dioxide acts as an etch stop layer. After removal of the ProTEK layer and cleaning of the sample, we perform vapour HF etching. The device side is here protected since it is electrostatically clamped to the chuck of the vapour HF tool. 


\subsection{Measurements}

To characterise the above device, we measure the shift of a spectral feature when a $5 \mathrm{ul}$ droplet containing $1.25 \mathrm{mg} / \mathrm{ml}$ bovine serum albumine (BSA) is added to a $5 \mathrm{ul}$ droplet of phosphate buffered saline (PBS) at pH 8 . This experiment is straightforward since no receptor molecules need to be applied to our sensor because BSA binds directly to silicon. Before starting the experiment, we want to ensure that our sensor is in fact hydrophilic. Although this should pose no real problem since the surface is still hydrophilic, we give our sensors a 10-minute oxygen plasma treatment. Not only does this reduce the contact angle to practically 0 degrees (i.e. full wetting), but it also acts as an extra cleaning step to remove organic contaminants.

A measurement is divided into several pump cycles: an underpressure of $10 \mathrm{kPa}$ is applied at the substrate side of the chip for $10 \mathrm{~s}$. After that, an overpressure of $10 \mathrm{kPa}$ is applied at the substrate side of the chip for the same number of seconds. The pressure is stabilised again (i.e. no pressure gradient) and the wavelength spectrum is measured 10 consecutive times. When this is done, a new pump cycle is started. Note that we do not perform measurements when there is either over- or underpressure. This is because the elasto-optic effect created by this pressure gradient would cause an additional shift of the spectral features which has to be decoupled from the biomeasurements.

Figure 6 shows the wavelength shift of a spectral dip at $1530 \mathrm{~nm}$ in function of time for the same biosensor in two situations (the sensor is reused after being cleaned using a Piranha solution): the dashed curve shows a measurement where the BSA droplet is introduced right before a new pump cycle starts and the solid curve shows a measurement where the BSA droplet is applied after pumping but before the wavelength measurements start, i.e. no subsequent pumping is performed to help the BSA reach the sensor. In both situations, before the BSA droplet is applied at $\mathrm{t}=210 \mathrm{~s}$, a pure PBS droplet is already present on the sensor.

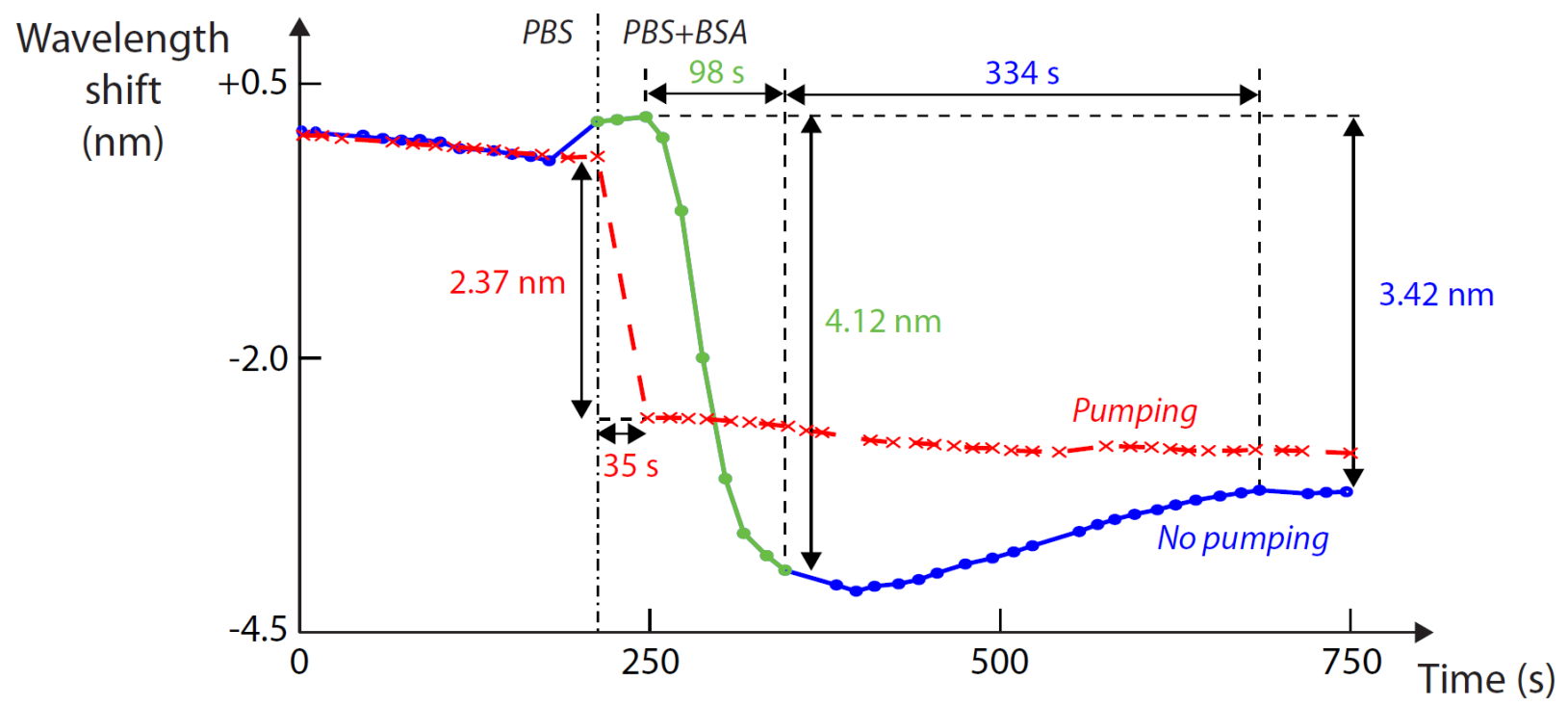

Figure 6. When pumping (red, dashed curve) the binding time can be decreased by a factor of 3, compared to no pumping (blue, solid curve). The green curve represents one measurement cycle of 10 data points.

When examining the binding event (i.e. transition from PBS to PBS+BSA), we notice a clear difference in the binding curves of both measurements. In the case where the BSA is applied after the pumping and before the 10 measurements of the current pump cycle, we see a typical binding curve (blue and green, solid curve) that starts off steeply and gradually reaches its peak value. In the other case, where the BSA is introduced and we immediately pump, we do not see this binding curve and immediately go into saturation (red, dashed curve). This is indeed the effect that we wanted to prove: by creating a flow path through the sensor (latter case), binding of the biomolecules occurs quicker than if we would have to wait for diffusion (former case). When quantitatively comparing both configurations, we see that the time it takes 
for binding with pumping is about $35 \mathrm{~s}$ (which is here limited by the pump duration and can be reduced upon optimisation) while the time it takes for the binding curve to reach its maximum is $98 \mathrm{~s}$, almost 3 times as slow.

\section{REFERENCES}

[1] S.K. Selvaraja, P. Jaenen, W. Bogaerts, D. Van Thourhout, P. Dumon, and R. Baets, “,” J. Lightwave Technol 27: 4076-4083 (2009).

[2] K. De Vos, J. Girones Molera, T. Claes, Y. De Koninck, S. Popelka, E. Schacht, R. Baets, P. Bienstman,” Multiplexed antibody detection with an array of silicon-on-insulator microring resonators," IEEE Phot Journal, 1(4): 225-235 (2009).

[3] D. Taillaert, P. Bienstman, R. Baets, "Compact efficient broadband grating coupler for silicon-on-insulator waveguides",Optics Letters, 29(23):.2749-2751 (2004).

[4] C. Lerma Arce, S. Van Put, A. Goes, E. Hallynck, P. Dubruel, K. Komorowska, P. Bienstman, "Reaction tubes: A new platform for silicon nanophotonic ring resonator sensors", Journal of Applied Physics, 115, p.044702, (2014).

[5] E. Hallynck, P. Bienstman, "Digital Microfluidics With Pressure-Based Actuation<, IEEE Photonics Technology Letters, 25(17), p.1656-1659 (2013). 